\title{
Influence of low energy argon plasma treatment on the moisture barrier performance of hot wire-CVD grown SiNx multilayers
}

\author{
Subimal Majee ${ }^{*}$, Maria Fátima Cerqueira2, Denis Tondelier1, Bernard Geffroy1,3, \\ Yvan Bonnassieux1, Pedro Alpuim2,4, and Jean Eric Bourée1 \\ 1Laboratoire de Physique des Interfaces et des Couches Minces, Ecole Polytechnique, CNRS UMR 7647, 91128 Palaiseau, \\ France \\ 2Centro de Física, Universidade do Minho, 4710-057 Braga, Portugal \\ 3Laboratoire de Chimie des Surfaces et Interfaces, IRAMIS/SPCSI CEA Saclay, 91191 Gif-sur-Yvette, France \\ 4INL-International Iberian Nanotechnology Laboratory, 4715-330 Braga, Portugal
}

The reliability and stability are key issues for the commercial utilization of organic photovoltaic devices based on flexible polymer substrates. To increase the shelf-lifetime of these devices, transparent moisture barriers of silicon nitride ( $\mathrm{SiNx}$ ) films are deposited at low temperature by hot wire CVD (HW-CVD) process. Instead of the conventional route based on organic/inorganic hybrid structures, this work defines a new route consisting in depositing multilayer stacks of SiNx thin films, each single layer being treated by argon plasma. The plasma treatment allows creating smoother surface and surface atom rearrangement. We define a critical thickness of the single layer film and focus our attention on the effect of increasing the number of SiNx single-layers on the barrier properties. A water vapor transmission rate (WVTR) of $2 \times 10^{-4} \mathrm{~g} /\left(\mathrm{m}^{2}\right.$ day) is reported for SiNx multilayer stack and a physical interpretation of the plasma treatment effect is given.

\section{Introduction}

The organic photovoltaic cells (OPV) fabricated on flexible plastic substrates have the potential to reduce the production costs by applying high throughput roll-to-roll coating processes, compared to the conventional process used for rigid solar cells.1) To achieve sufficient shelf-lifetime for commercial applications, OPV cells require encapsulation with a low permeability barrier material toward atmospheric oxidizing agents, especially low transmission rates in water vapor.2) Whereas sensitive food products can be protected with a single polymer film, organic solar cells need, in order to work during several thousands of hours,2-4) permeation barriers composed of complex layer stacks. A typical water vapor transmission rate (WVTR) as low as $10^{-4} \mathrm{~g} /\left(\mathrm{m}^{2}\right.$ day) is required. The single-layers of transparent materials such as silica, silicon nitride or alumina, often used as barrier layers, have pinholes through which water molecules can diffuse up to the organic device surface and thus deteriorate their performance.5-9) Due to that reason, more and more research has been focused on minimizing the propagation of defects by developing a multilayer stack.1) Up to now the conventional approach consisted in depositing alternate organic/ inorganic layers in order to minimize the stress inside the inorganic films4,9-12) and in depositing alternate inorganic/ inorganic layers in order to minimize the pinhole propagation. 13) In the present work, we focus our attention on a new process consisting in depositing multilayer stacks of $\mathrm{SiN} x$ single-layers, each single-layer being surface-treated by argon plasma, except the last one. We have chosen hot wire chemical vapor deposition (HW-CVD) method to deposit the $\mathrm{SiNx}$ films at low temperature because of the lower hydrogen content inside the film and the slightly higher density compared to the conventional material obtained by plasma assisted CVD techniques.9,13-19)

The idea behind the use of the argon plasma surface treatment between two successive SiNx single-layers (with a relatively high porosity caused by the low temperature deposition process) is to hinder the propagation of the pinholes from one single-layer to the other. The bombardment of low energy $(<100$ $\mathrm{eV}$ ) argon ions on the surface of the $\mathrm{SiN}_{x}$ thin films may smoothen the surface19) and rearrange the surface atoms,20) thus decoupling the structural defects in consecutive single-layers. There are several parameters that can be varied during the Ar ionic treatment (e.g., energy of ions, the flux of ions, frequency of plasma generation and duration of treatment). In a previous work, we have studied the effect of high frequency $(13.56 \mathrm{MHz})$ and low energy $(<100 \mathrm{eV})$ ionic treatment on the barrier properties of SiNx double-layers (total thickness: $100 \mathrm{~nm}$ ), while varying the treatment duration in the range from 2 to $15 \mathrm{~min}$ and found an optimum treatment time of 8 min above which there was no significant barrier improvement.19) In the present study, we use these optimized conditions for the argon plasma surface treatment and study the effect of increasing the number of single-layers on the permeation barrier properties. In order to assess the permeation barrier properties of these multilayers, we measure the WVTR values using the electrical calcium degradation test method at ambient atmospheric conditions.19,21-23). 


\section{Experimental methods}

In order to deposit SiNx thin films by HW-CVD process, the precursor gases (ammonia, silane, and hydrogen) are introduced into a vacuum chamber, and a single coil shaped tantalum filament is heated up to $2000{ }^{\circ} \mathrm{C}$. The filament acts as a catalyst, providing thereby the surface for heterogeneous thermal decomposition of the gases into radical species, which are the precursors for the amorphous SiNx film growth. The filament to substrate distance is $7.5 \mathrm{~cm}$ and the substrate temperature $\left(\mathrm{T}_{\mathrm{sub}}\right)$ is fixed at $100{ }^{\circ} \mathrm{C}$ in order to be compatible with the glass transition temperature $\left(\mathrm{Tg}_{\mathrm{g}}\right)$ of the plastic substrates.9,13,18,19) Poly(ethylene terephthalate) (PET; Melinex ST 504, 175 $\mu$ m thick) is used as the plastic substrate, because it is highly flexible, widely used and inexpensive. Single and multilayers of SiNx thin films are obtained using optimized deposition conditions described previously under a working pressure of $25 \mathrm{mTorr}$.19) Due to the increase of the substrate temperature with time caused by the heated filament, the single-layer thickness is limited in the range of 50-60 nm. The Ar-plasma treatment between successive SiNx singlelayers is carried out in a glow discharge chamber, the sequence of $\mathrm{SiN} \times$ deposition and Ar treatment being performed without breaking the vacuum by moving the sample between the two twin chambers connected by a gate valve. In the RF (13.56MHz) chamber the sample is clamped to the top electrode which is heated up to $100{ }^{\circ} \mathrm{C}$ and the bottom electrode is the RF-powered electrode. The working pressure is $50 \mathrm{mTorr}$ and the power density is $350 \mathrm{~mW} / \mathrm{cm} 2$. The optimum treatment duration $(8 \mathrm{~min})$ is chosen, which produces the minimum WVTR as described previously.19)

The films are characterized by X-ray diffractometry as described previously.19) To understand the possible effects of the treatment on the thin films, the roughness of the films deposited on very flat crystalline silicon wafers is measured using atomic force microscopy (AFM) in tapping mode and analyzed using the instrument software. The refractive index, $n$, and thickness, $d$, of the films deposited on 510- $\mu$ m-thick crystalline silicon wafers is measured using the phase modulated spectroscopic ellipsometer Uvisel (Horiba Jobin- Yvon) in the 1.5-5.0 eV photon energy range. The measured data is fitted with the classical Lorentz dielectric model.18,19) To check the compatibility of these films with the utilization in real OPV devices, the reflectance $(R)$ and the transmittance $(T)$ curves of films on plastic substrates are measured using Perkin Elmer Integrating Sphere.

The permeation rate and the lag-time of diffusion of water vapor through the barrier coated plastic are evaluated using the electrical calcium degradation test under ambient atmospheric conditions.19,21-23) For the $\mathrm{Ca}$ degradation measurement, the amount of $\mathrm{Ca}$ is fixed for all the measurements, with an area of 1.13 $\mathrm{cm}^{2}$ and a thickness of $100 \mathrm{~nm}$. The detailed description and the schematic view of the measurement are described elsewhere.19) Water molecules in the environmental moisture, after diffusing through the pinholes and defects inside the barrier, react with the metallic $\mathrm{Ca}$ sensor and transform it into insulating and transparent $\mathrm{Ca}(\mathrm{OH})_{2}$ film, whose resistivity increases accordingly. The $\mathrm{Ca}$ sensor resistance is measured using a four point probe technique. The WVTR value is deduced from the average decrease of conductance with time of the Ca layer (dG/dt), expressing its transformation under the action of water molecules:

$$
W V R T\left[g / \text { day. } m^{2}\right]=-N \delta_{C a} \rho_{C a} \frac{M\left(H_{2} O\right)}{M_{C a}} \frac{l}{w} \frac{d(G)}{d t} \frac{\operatorname{area}(\mathrm{Ca})}{\text { area }(\text { window })}
$$

where $\mathrm{N}$ is the molar equivalent of the degradation reaction which is assumed as $\mathrm{N}=2$ from the chemical reaction of $\mathrm{Ca}$ with water $\left[\mathrm{Ca}+2 \mathrm{H}_{2} \mathrm{O}^{1} / 4 \mathrm{Ca}(\mathrm{OH})_{2}+\mathrm{H}_{2}\right], \mathrm{M}$ is the molar mass of the reactive elements, $\delta \mathrm{Ca}$ is the density of calcium $\left(1.55 \mathrm{~g} / \mathrm{cm}^{3}\right), \rho \mathrm{Ca}$ is the calcium resistivity $\left(9 \times 10^{-8} \Omega \mathrm{m}\right), \mathrm{I}$ and $\mathrm{W}$ are the effective length and width of $\mathrm{Ca}$ layer, respectively, and $\mathrm{G}$ is the conductance. From the geometry of our setup, the value of Area (Ca)/Area (window) is taken to be unity.19)

We first measure the effect of SiNx single-layer coating thickness (from 10 to $60 \mathrm{~nm}$ ) on the permeation barrier properties and thus define a critical thickness. We then assess the barrier properties of multi-layer structures with Ar treatment, while considering the critical thickness of the single-layer as a fixed parameter.

\section{Results and discussion}

\subsection{Determination of critical thickness}

Figure 1 shows the evolution of normalized conductance of Ca sensors, deposited on PET substrates whose back side has been coated with $\mathrm{SiN} \times$ single-layers with thickness ranging from 10 to $60 \mathrm{~nm}$. Bare plastic is used as the reference for the calcium degradation test. The degradation test has been performed on 10 uncoated PET substrates to evaluate the reproducibility of the measurement. The WVTR value 
assessed from the conductance evolution curve shows that the uncoated PET substrate has a WVTR of $0.17+/-0.05 \mathrm{~g} /\left(\mathrm{m}^{2}\right.$ day), whereas the WVTR measured for glass substrate (not shown here) is aprox. $10^{-6}$ $\mathrm{g} /\left(\mathrm{m}^{2}\right.$ day $\left.) .19\right)$

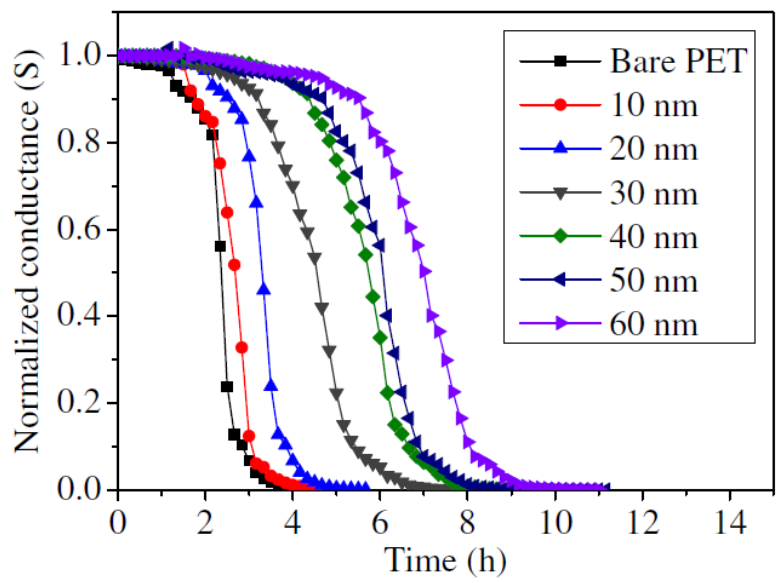

Fig. 1. (Color online) Evolution of normalized conductance of Ca sensor deposited on PET coated with single-layers of SiNx thin films with different thicknesses. The uncoated PET is shown as reference.

With the increase of the SiNx single-layer thickness, the minimum time taken by the water molecules to permeate through the whole barrier thickness including PET substrate ("lag-time"), is increased as can be seen in Fig. 2. As seen in Fig. 3, the barrier performance assessed by the decrease of the WVTR value also improves with the increase of $\mathrm{SiN}_{x}$ single-layer coating thickness $(\mathrm{d})$. The curve can be divided into three regions .24)

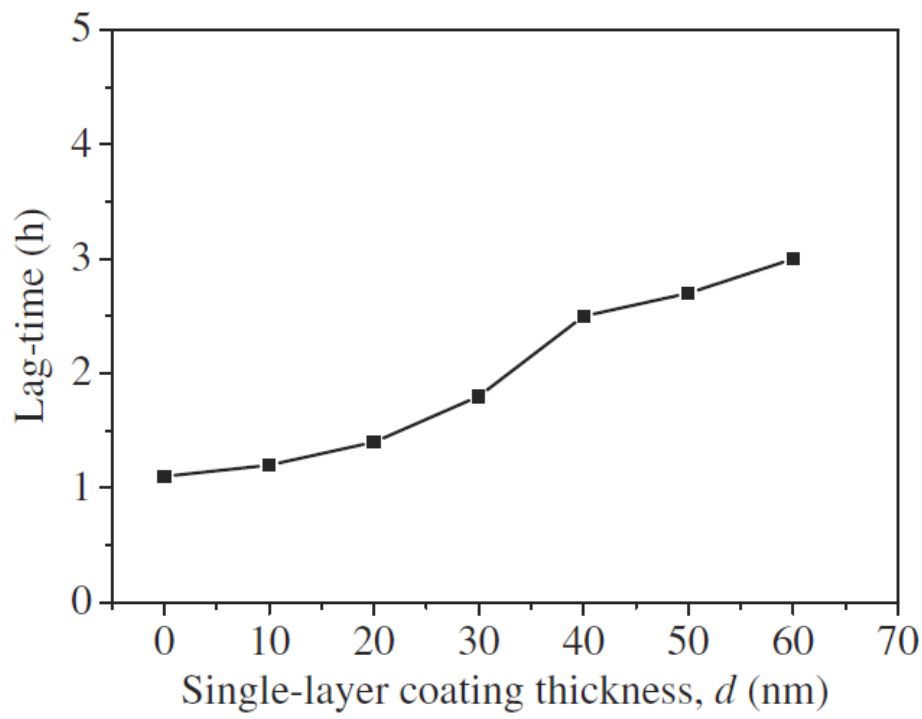

Fig. 2. Variation of "lag-time" with SiNx single-layer coating thickness. 


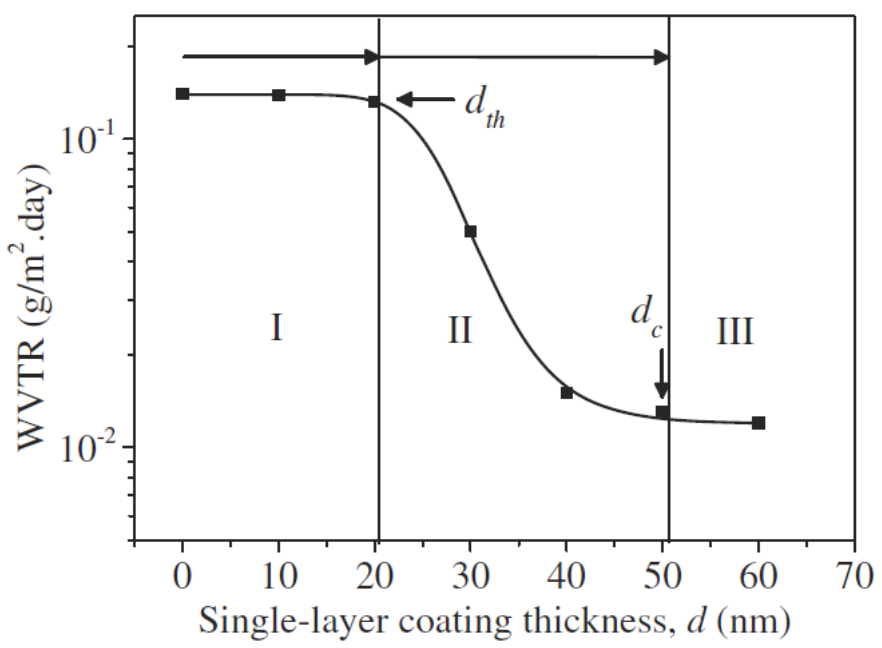

Fig. 3. Effect of $\mathrm{SiN}_{x}$ single-layer coating thickness on WVTR values.

The first one, from 0 to $20 \mathrm{~nm}$, corresponds to the "threshold thickness ( $d_{\text {th)" }}$ region, below which there is no barrier effect and over which $\left(d>d_{\text {th }}\right)$ the permeation rate drops steeply until it stabilizes at a critical thickness $\left(d_{c}\right)$ value (about 40 to $50 \mathrm{~nm}$ in this case). For, $d>d_{c}$, no further significant decrease in WVTR is observed, the stabilized value being aprox. $1 \times 10^{-2} \mathrm{~g} /\left(\mathrm{m}^{2}\right.$ day). From this first measurement, we define the critical thickness as the maximum single-layer thickness producing the maximum WVTR reduction. Based only on these WVTR results, it is expected that the stack of several SiNx single-layers, each having a thickness equal to or greater than the critical thickness $\left(d_{c}\right)$, will be very effective in reducing the overall WVTR value and thereby in improving the overall performance of the permeation barrier.

The average surface roughness evolves similarly to the WVTR versus the single-layer thickness. Figure 4 shows the decrease in surface roughness from 55 to $23 \mathrm{~nm}$ when the PET substrate is covered by a SiNx layer of thickness at least equal to the critical thickness. It should be noted that the roughness of PET foil increases from 1.35 to $55 \mathrm{~nm}$ after exposure to the hot Ta filament, which explains the high initial value of roughness in Fig. 4.19)

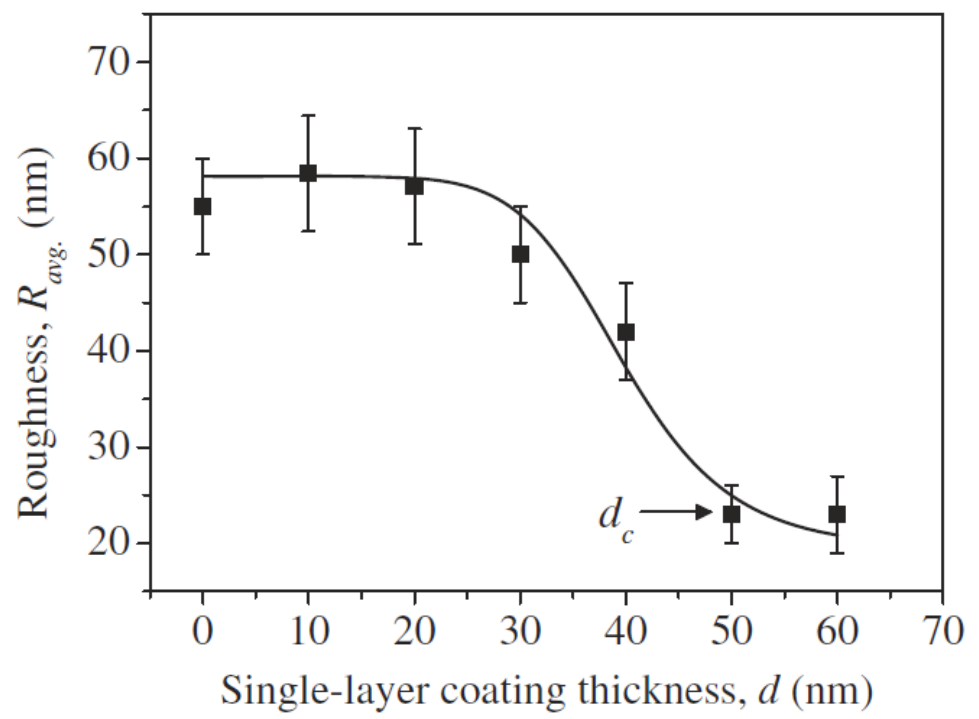

Fig. 4. Variation of average roughness of $\mathrm{SiN} x$ single-layers coated on PET substrates with the coating thickness.

The improvement in barrier performance is limited mainly by pinholes or nanometer-to-micron sized structural defects, which can favor the diffusion of water molecules from the underlying SiNx film. So we looked for a technique which could drastically modify the sub-surface structure near the interface without modifying the bulk layer structure. We chose the technique of low energy argon plasma surfasse treatment (energy lower than sputtering energy). 


\subsection{Effect of Ar-plasma treatment on morphology}

Characterizations are undertaken in order to observe the effect of the low energy Ar plasma surface treatment on the film surface. We performed AFM measurements on as-grown and plasma-treated $\mathrm{SiNx}$ $(50 \mathrm{~nm})$ thin films deposited on crystalline silicon wafers, shown in Fig. 5, in order to verify the smoothening of the surface done by plasma treatment. 19) The as deposited SiNx films observed in Fig. 5(a) exhibits a comparatively rough surface with $2.67 \mathrm{~nm}$ peaks on the surface. After 8 min treatment of Ar plasma, the film shows a flat surface [Fig. 5(b)] with approx. 85\% reduction of the root mean squared $(\mathrm{rms})$ roughness $(0.39 \mathrm{~nm})$ compared to untreated sample $(2.67 \mathrm{~nm})$. From this result it is clear that ion treatment leads to a surface modification. The effect of surface smoothening may be due to the exchange of energy between the impinging ions with sufficient energy $(<100 \mathrm{eV})$ and the surface atoms vibrating around their equilibrium positions. Modification of the underlying $\mathrm{SiN}_{\mathrm{x}}$ layer could help in hindering the propagation of any structural defects. When these Ar-treated SiNx single-layers are stacked to form a multilayer structure, then this should logically improve the barrier performance of the whole structure.

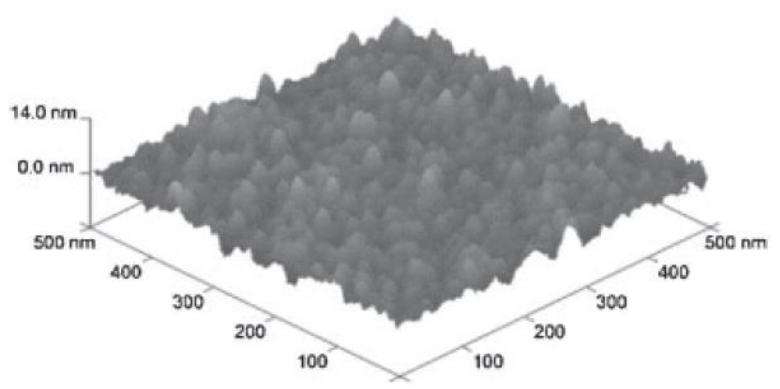

(a)

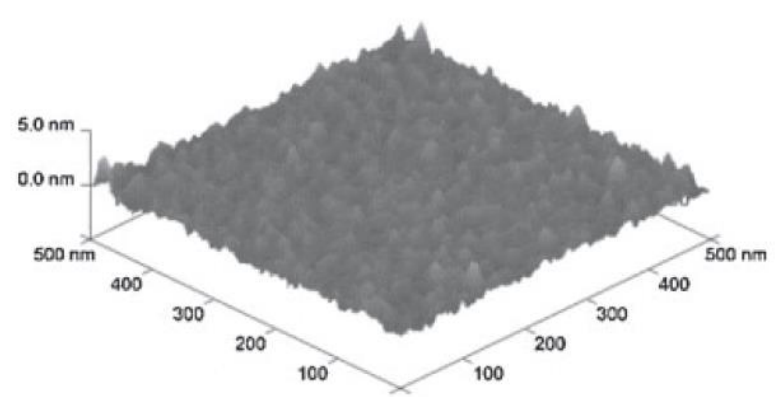

(b)

Fig. 5. Three-dimensional AFM images of the surface $\left(500 \times 500 \mathrm{~nm}^{2}\right)$ of SiNx layer (a) without and (b) with Ar-plasma treatment deposited on c-Si wafers. The plasma treated sample shows an rms roughness reduction of $85 \%$ compared to untreated surface.

\subsection{Effect of Ar-plasma treatment on optical properties}

Figure 6 shows the optical data consisting of reflectance and transmittance measurements of the multilayer structure (stack of five $\mathrm{SiNx}$ single-layers, each separated by $8 \mathrm{~min}$ of $\mathrm{Ar}+$ plasma treatment) deposited on PET substrates. The transmittance curve indicates that the multi-layer structure is highly transparent (approx. 80\%) in the visible region. It can also be seen from Fig. 6 that reflectance and transmittance of this multi-layer structure add to almost $100 \%$, meaning that no absorption takes place in the layers and the barrier structure can be perfectly used as a window layer for OPV cells. Slight absorption below $400 \mathrm{~nm}$ wavelength is due to the plastic substrate itself. 


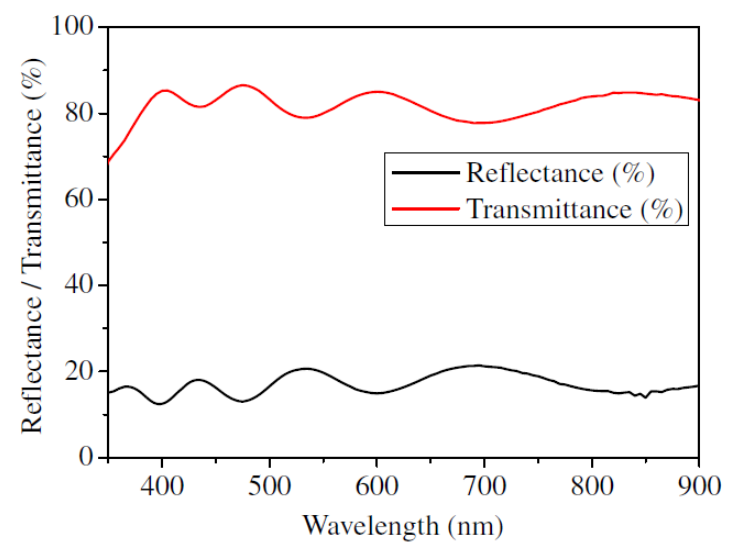

Fig. 6. (Color online) Reflection and transmission spectra of $\mathrm{SiN} \times$ multilayer (stack of five $\mathrm{SiN} \times$ single-layers each separated by Artreatment deposited on PET substrate).

\subsection{Effect of Ar-plasma treatment on permeation barrier properties}

The evolutions of normalized conductance of the Ca sensors deposited on such multi-barrier coated PET substrates are shown in Fig. 7. Slight kinks are observable in the traces, which are probably due to the inhomogeneous oxidation of the calcium surface caused by the in-homogeneities inside the barrier layer and the PET substrate. When the number of $\mathrm{SiNx}$ single layers increases, the number of interfaces between $\mathrm{SiNx}$ films treated with argon plasma increases as well. The water molecules face a complex diffusion path when passing from one $\mathrm{SiN}_{x}$ single-layer to the other through the intermediate treated $\mathrm{SiN}_{\mathrm{x}}$ interfaces. Due to that reason and to the increase in total thickness of the coating, the minimum time taken by the water molecules to reach the $\mathrm{Ca}$ sensor which is at the opposite side of the coated PET substrates ("lag-time"), increases with increasing number of stacked SiNx single-layers (Fig. 8). A maximum lagtime of $77 \mathrm{~h}$ is achieved with five stacked $\mathrm{SiN} x$ single-layers, each layer being separated from the next one by the Ar-treatment.

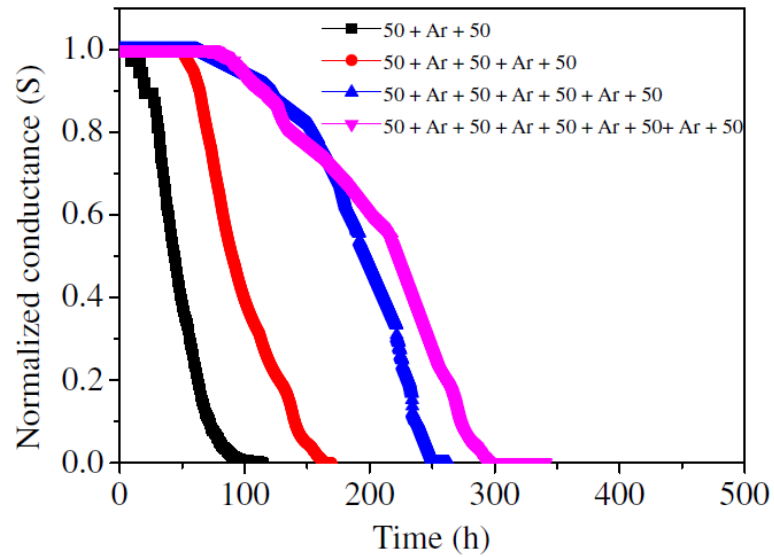

Fig. 7. (Color online) Evolution of normalized conductance of Ca sensor deposited on PET coated with multi-layers of SiNx films treated by argon plasma

The WVTR values also decreases monotonically with increasing number of layers (see Fig. 9) reaching a minimum value of aprox. $2 \times 10^{-4} \mathrm{~g} /\left(\mathrm{m}^{2}\right.$ day $)$. This tendency suggests that if the number of layers is further increased, then the barrier properties will be better. But the increased thickness will decrease the optical transparency and the flexibility of the whole structure. Obviously, if the mean energy of the Ar+ was higher, the treatment duration would be less than $8 \mathrm{~min}$, but the higher ion energy would also promote atomic sputtering and probably damage the barrier properties of the interfaces. 


\subsection{Physical interpretation}

The modification of roughness and barrier properties observed in the previous sections can be interpreted taking into account the main physical parameters of the Ar plasma. First of all, the drastic decrease of the rms roughness under the Ar plasma treatment observed in Fig. 5 can be interpreted as a minimization of surface energy (i.e., minimization of effective surface for a constant surface tension) under the action of the low energy $(<100 \mathrm{eV})$ Ar ion flux impinging on the SiNx film surface. Such ions striking a material can penetrate to a certain depth within a depth distribution and rearrange the atoms locally in order to impede the spreading of structural defects. The depth distribution depends on the energy and the atomic mass of these ions related to the atomic mass of target atoms. During their travel through the material, energy is transferred to the near environment. Ions can transfer energy in a pure mechanical way by nuclear interaction (nuclear stopping power), through phonons (atomic vibrations), but can also loose energy to electrons (electronic stopping power). These mechanisms will cause rearrangement of the neighboring bulk atoms, initiating an atomic collision cascade.25) As a result, target atoms will be relocated from their original position, causing either damage to the interface structure, and/or densification of the interface up to few $\mathrm{nm}$ in depth. The argon ion bombardment on the surface must be such that it disrupts the atomic distribution to a few $\mathrm{nm}$ in depth, disturbing the continuity of a structural defect through this interface but it cannot significantly damage the interface. For the conditions used in this work, the argon ion energy after being accelerated through the plasma sheath potential is $<100 \mathrm{eV}$. The bombardment by argon ions with this energy promotes the $\mathrm{SiNx}$ single-layer surface smoothening and possibly the densification of the surface to a few $\mathrm{nm}$ in depth by sufficient rearranging of the interface atoms (what should be confirmed by X-ray reflectivity measurement in a future work).26,27)

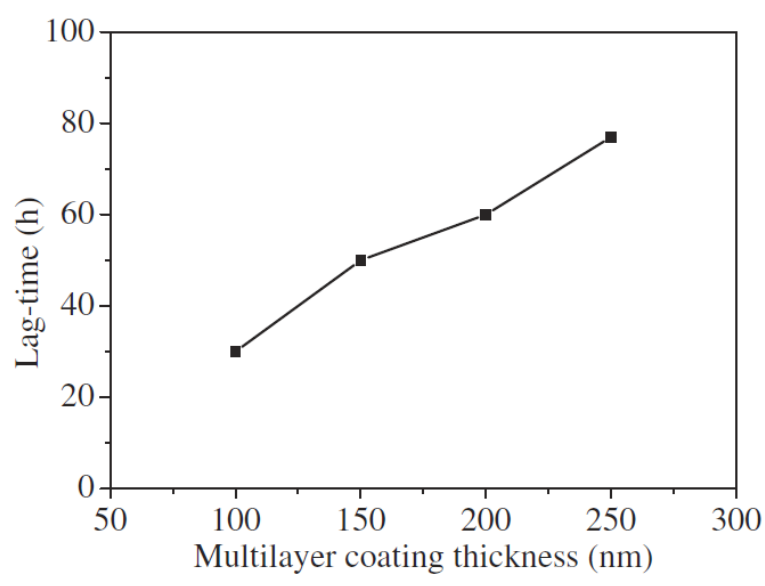

Fig. 8. Variation of "lag-time" with SiNx multi-layer coating thickness (Ar treated).

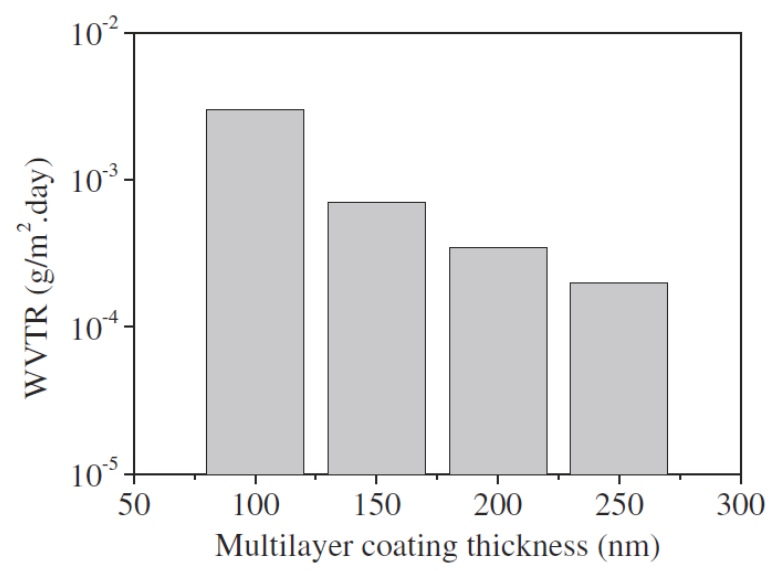

Fig. 9. Variation of WVTR with SiNx multi-layer coating thickness (Ar treated).

\section{Conclusions}

In this paper, using the HW-CVD technique, based on silane and ammonia gaseous mixtures diluted in hydrogen at a substrate temperature of $100{ }^{\circ} \mathrm{C}$ and after an optimization taking into account the different 
growth parameters, we have deposited device quality transparent and stoichiometric silicon nitride thin films which can be used as moisture barrier layers for encapsulation of flexible organic photovoltaic cells. We have observed the evolution of the lag-time and WVTR value with the increasing SiNx single-layer thickness and defined a critical thickness of $50 \mathrm{~nm}$, above which both quantities do not improve any more. The permeation rate is further reduced using a multilayer of $\mathrm{SiN}_{x}$ films (where the critical thickness is used as a fixed parameter) and an Ar-plasma treatment between each successive singlelayer. Using Ar ions with a power density of $350 \mathrm{~mW} / \mathrm{cm}^{2}$ and an average ion energy of $<100 \mathrm{eV}$ during $8 \mathrm{~min}$, the lagtime is increased up to $77 \mathrm{~h}$ and the permeation rate is reduced down to $2 \times 10^{-4} \mathrm{~g} /\left(\mathrm{m}^{2}\right.$ day $)$. Surface roughness decrease (smoothening of the surface) under the action of the $\mathrm{Ar}+$ ions has been observed and explained as a consequence of the minimization of surface energy. These low energy ions induce a structural modification at each interface, leading to a tortuous permeation path for the water molecules through the barriers. Thus the low value of WVTR $=2 \times 10^{-4} \mathrm{~g} /\left(\mathrm{m}^{2}\right.$ day $)$ observed in the SiNx multilayers treated by Ar plasma should be sufficient for flexible organic photovoltaic cells to have a long shelf-lifetime.

\section{Acknowledgements}

The authors would like to thank Cyril Jadaud for help in mechanical design, Dr. J. C. Vanel for help in electrical characterizations, and Dr. Hassina Derbal Habak for help in optical characterizations used in this study. The first author (S.M.) acknowledges the financial support from Direction des Relations Extérieures, Ecole Polytechnique during his thesis. This study has been supported by PICS (FrenchPortuguese No. 5336) project.

1) J. Fahlteich, M. Fahland, W. Schönberger, and N. Schiller, Thin Solid Films 517, 3075 (2009).

2) S. Cros, R. de Bettignies, S. Berson, S. Bailly, P. Maisse, N. Lemaitre, and S. Guillerez, Sol. Energy Mater. Sol. Cells 95, S65 (2011).

3) J. A. Hauch, P. Schilinsky, S. A. Choulis, S. Rajoelson, and C. J. Brabec, Appl. Phys. Lett. 93, 103306 (2008).

4) H. Nakayama and M. Ito, Thin Solid Films 519, 4483 (2011).

5) W. Prins and J. J. Hermans, J. Phys. Chem. 63, 716 (1959)

6) T. A. Beu and P.-V. Mercea, Mater. Chem. Phys. 26, 309 (1990).

7) A. S. da Silva Sobrinho, G. Czeremuszkin, M. Latrèche, and M. R. Wertheimer, J. Vac. Sci. Technol. A 18, 149 (2000).

8) M. S. Weaver, L. A. Michalski, K. Rajan, M. A. Rothman, J. A. Silvernail, J. J. Brown, P. E. Burrows, G. L. Graff, M. E. Gross, P. M. Martin, M. Hall, E. Mast, C. Bonham, W. Bennett, and M. Zumhoff, Appl. Phys. Lett. 81, 2929 (2002).

9) D. Spee, K. van der Werf, J. Rath, and R. Schropp, Phys. Status Solidi: Rapid Res. Lett. 6, 151 (2012).

10) J. Greener, K. C. Ng, K. M. Vaeth, and T. M. Smith, J. Appl. Polym. Sci. 106, 3534 (2007).

11) T. W. Kim, M. Yan, A. G. Erlat, P. A. McConnelee, M. Pellow, J. Deluca, T. P. Feist, A. R. Duggal, and M. Schaepkens, J. Vac. Sci. Technol. A 23, 971 (2005).

12) V. Verlaan, R. Bakker, C. H. M. van der Werf, Z. S. Houweling, Y. Mai, J. K. Rath, and R. E. I. Schropp, Surf. Coatings Technol. 201, 9285 (2007).

13) Y. Ogawa, K. Ohdaira, T. Oyaidu, and H. Matsumura, Thin Solid Films 516, 611 (2008).

14) H. Matsumura, J. Appl. Phys. 66, 3612 (1989)

15) H. Matsumura, H. Umemoto, A. Izumi, and A. Masuda, Thin Solid Films 430, 7 (2003)

16) A. Masuda, M. Totsuka, T. Oku, R. Hattori, and H. Matsumura, Vacuum 74, 525 (2004).

17) A. Heya, T. Niki, Y. Yonezawa, T. Minamikawa, S. Muroi, A. Izumi, A. Masuda, H. Umemoto, and H. Matsumura, Jpn. J. Appl. Phys. 43, L1362 (2004)

18) P. Alpuim, L. M. Gonçalves, E. S. Marins, T. M. R. Viseu, S. Ferdov, and J. E. Bourée, Thin Solid Films 517, 3503 (2009).

19) S. Majee, M. F. Cerqueira, D. Tondelier, B. Geffroy, Y. Bonnassieux, P. Alpuim, and J. E. Bourée, Surf. Coatings Technol. 235, 361 (2013).

20) J. Ubrig, S. Martin, S. Cros, and J.-E. Bouree, J. Phys.: Conf. Ser. 100, 082030 (2008).

21) R. Paetzold, A. Winnacker, D. Henseler, V. Cesari, and K. Heuser, Rev. Sci. Instrum. 74, 5147 (2003).

22) M. O. Reese, A. A. Dameron, and M. D. Kempe, Rev. Sci. Instrum. 82, 085101 (2011).

23) S. Schubert, H. Klumbies, L. Müller-Meskamp, and K. Leo, Rev. Sci. Instrum. 82, 094101 (2011).

24) Y. Leterrier, Prog. Mater. Sci. 48, 1 (2003).

25) P. Sigmund, in Radiation Damage Processes in Materials, ed. C. H. S. Dupuy (Noordhoff, Leyden, 1975) p. 3

26) J. Cuomo, S. M. Rossnagel, and H. R. Kaufman, Handbook of Ion Beam Processing Technology (Noyes Publications, Park Ridge, NJ, 1989) p. 180

27) R. Vernhes, A. Amassian, J. E. Klemberg-Sapieha, and L. Martinu, J. Appl. Phys. 99, 114315 (2006). 